\title{
Mechanical Prosthetic Heart Valve Thrombosis in a Patient Receiving Rivaroxaban
}

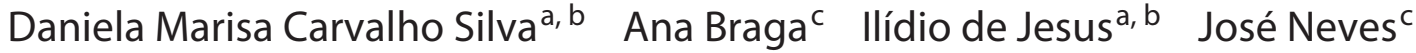 \\ ${ }^{a}$ Cardiology Department, Centro Hospitalar e Universitário do Algarve, Faro, Portugal; ${ }^{b}$ Algarve Biomedical Center, \\ Faro, Portugal; ${ }^{C}$ Cardiothoracic Department, Hospital de Santa Cruz, Centro Hospitalar de Lisboa Ocidental, \\ Carnaxide, Portugal
}

\section{Established Facts}

- Direct oral anticoagulants are not indicated in patients with mechanical valve prostheses.

- Scientific evidence of the use of these drugs in mechanical prosthetic valve prostheses is scarce and points to an excess of thromboembolic and hemorrhagic phenomena.

- Valve replacement surgery is the therapy of choice in obstructive thrombosis of mechanical prosthesis, being a high-risk intervention.

\section{Novel Insights}

- There have been no previously described cases of mechanical valve prosthesis thrombosis in the mitral position associated with the use of rivaroxaban.

\section{Keywords}

Mechanical prosthesis - Prosthetic heart valve .

Thrombosis · Direct oral anticoagulants · Rivaroxaban

\section{Abstract}

Direct oral anticoagulants are not approved for use in patients with mechanical valves. When used to replace vitamin $\mathrm{K}$ antagonists, they may cause catastrophic consequences. The authors describe the case of a patient who, after discontinuation of warfarin and introduction of rivaroxaban, developed thrombosis of his mechanical mitral prosthesis.

(c) 2019 S. Karger AG, Basel

\section{Introduction}

Direct oral anticoagulants are not approved for use in patients with mechanical valve prostheses [1]. Unfortunately, many times, direct anticoagulants are still prescribed in these cases.

\section{Case Report and Case Presentation}

The authors report the case of a 67-year-old male with a history of dyslipidemia, former smoking, epilepsy, and rheumatic fever in childhood. Twenty years ago, he was

\section{KARGER}

(c) 2019 S. Karger AG, Basel

E-Mail karger@karger.com

www.karger.com/crd
Daniela Marisa Carvalho Silva

Cardiology Department, Centro Hospitalar e Universitário do Algarve Rua Leão Penedo

PT-8000-386 Faro (Portugal)

E-Mail danielacarvalhosilva@gmail.com 
Fig. 1. TTE with pulsed Doppler of the transprosthetic flow, showing an increase in the mean gradient and a decrease in the effective valve area.



diagnosed with rheumatic mitral stenosis and underwent percutaneous mitral valvuloplasty. Six years later, he was admitted with a diagnosis of non-ST-segment elevation myocardial infarction and underwent coronary angiography, having performed angioplasty of the anterior descending artery and ramus with stents. At that time, he took medication that included acetylsalicylic acid and statin. The statin was discontinued a few months later by his attending physician due to intense myalgias.

Over time, an increase in mitral transvalvular gradients was observed. Eleven years ago, episodes of atrial fibrillation have been documented, and therefore the patient has begun therapy for heart rate control and oral hypocoagulation with warfarin, with a target international normalized ratio (INR) of 2-3. Acetylsalicylic acid was suspended.

Ten years ago, the patient underwent mitral valvular replacement surgery with the St. Jude mechanical prosthesis 29 and closure of the left atrial appendage without intercurrences. Since then, he has taken warfarin. He reports that the INR target was 3 (range: $2.5-3.5$ ), which was achieved most of the time.

Six months ago, he had an appointment with a private general practitioner who recommended suspension of warfarin. The patient was then prescribed rivaroxaban 20 mg once daily.

He remained asymptomatic until 1 week before the latest hospital admission. At that time point, he reported an episode of epigastric pain lasting several hours during the night, which relieved spontaneously. In the next morning, he refers to onset of dyspnea for minimal efforts.

He denied fever, anorexia, weight loss, cutaneous or vascular changes, orthopnea, paroxysmal nocturnal dyspnea, palpitations, syncope, edemas, and changes in muscle strength or sensitivity.

In addition to rivaroxaban, he was taking telmisartan $40 \mathrm{mg}$ i.d., hydrochlorothiazide $12.5 \mathrm{mg}$ i.d., digoxin $0.125 \mathrm{mg}$ i.d. and carbamazepine $400 \mathrm{mg}$ i.d. The patient refers strict compliance to drug therapy.

Given the persistence of the symptoms, he turned to a private cardiologist. The electrocardiogram showed sinus rhythm and 1st-degree atrioventricular block. A transthoracic echocardiogram (TTE) was performed, which revealed good left ventricular function, with a marked increase in the gradients of mechanical mitral prosthesis (mean gradient of $24 \mathrm{~mm} \mathrm{Hg}$ ), reason for which he was referred to our hospital and admitted to the cardiology department.

At examination, he was hemodynamically stable, with a blood pressure of $114 / 70 \mathrm{~mm} \mathrm{Hg}$ and a heart rate of 90 bpm. Cardiac auscultation revealed a decreased intensity of the closing click of the mitral prosthesis and a soft diastolic rumble. Pulmonary auscultation showed vesicular murmur, without adventitious sounds, namely crackles. The presence of peripheral edema was not observed.

The analytical evaluation showed elevation of C-reactive protein (CRP: $159 \mathrm{mg} / \mathrm{L}$; normal range $[\mathrm{NR}]:<5)$ and 
Fig. 2. TEE showing pulsed Doppler of the transprosthetic flow, corroborating the findings of the TTE.
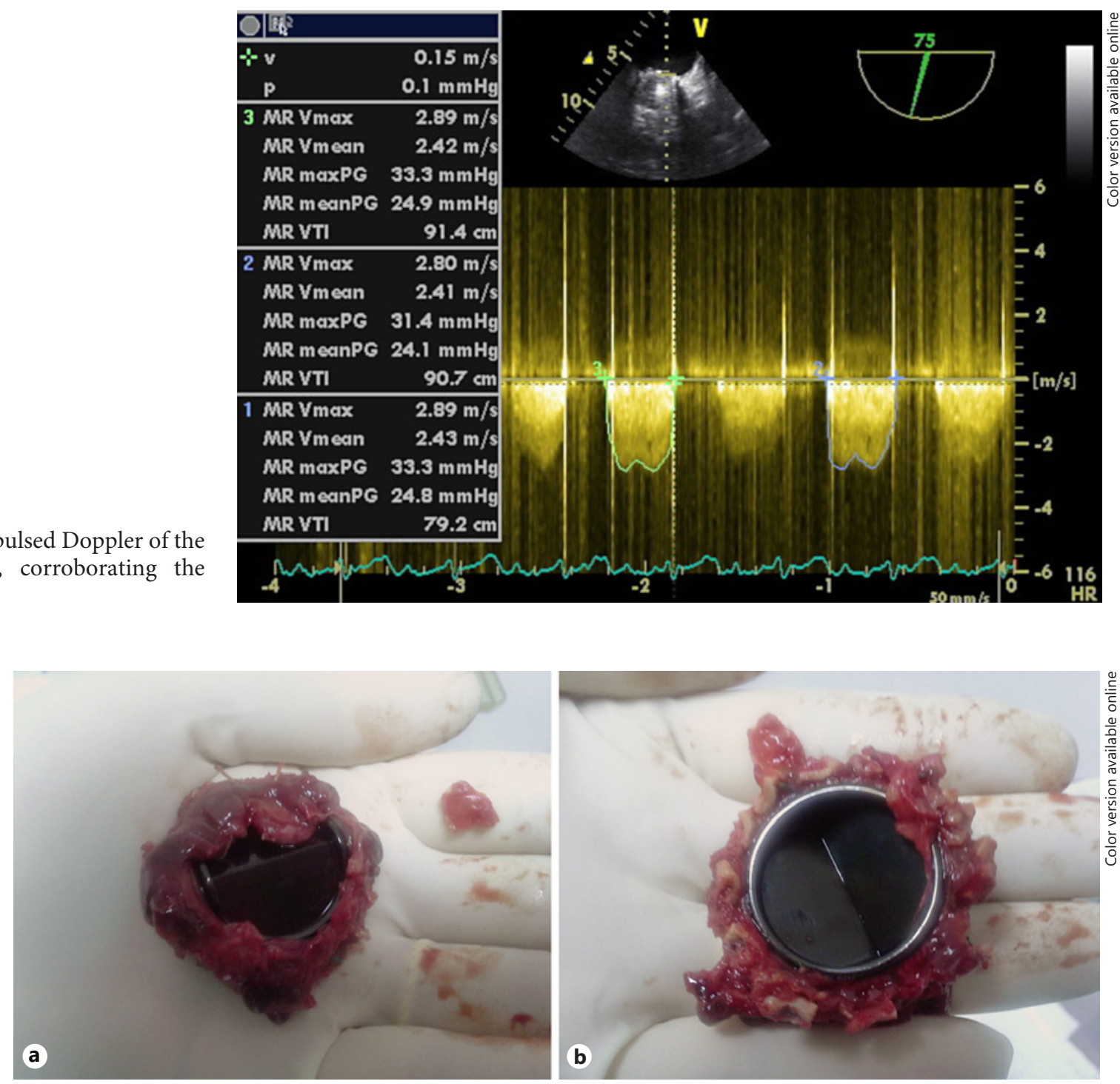

Fig. 3. a, b Images of the mechanical valvular prosthesis St. Jude 29 after its surgical resection.

no alterations in the hemogram, ionogram, or renal function. troponin I was also within the NR.

A new TTE revealed a mechanical prosthesis in the mitral position with restriction of the disc opening (online supplementary video $1 \mathrm{a}-\mathrm{d}$; for all online suppl. material, see http://www.karger.com), conditioning in an average gradient of $22.8 \mathrm{~mm} \mathrm{Hg}$ and a functional area of 0.6 $\mathrm{cm}^{2}$ (Fig. 1), suggestive of severe prosthetic dysfunction. The estimated pulmonary artery pressure was $40 \mathrm{~mm} \mathrm{Hg}$ (suggestive of mild pulmonary hypertension).

The patient underwent fluoroscopy, which showed severe dysfunction of mechanical mitral prosthesis due to immobility of one of the discs and severe limitation of the mobility of the second disc (suppl. video 2a, b). Coronary angiography revealed single vessel disease, with a plaque located on the proximal segment of the anterior descending artery conditioning 75-90\% luminal stenosis, proximal to previously implanted stents. Carotid angiography did not show significant atherosclerotic disease.

On the following day, he underwent transesophageal echocardiography (TEE), which corroborated the previously described findings (suppl. video 3a-d; Fig. 2).

The patient collected 3 sets of blood cultures and a urine culture, which turned out to be negative. CRP declined spontaneously without antibiotic therapy. 
Fig. 4. TTE with pulsed Doppler of the On-X 27/29 transprosthetic flow, showing normal velocities.

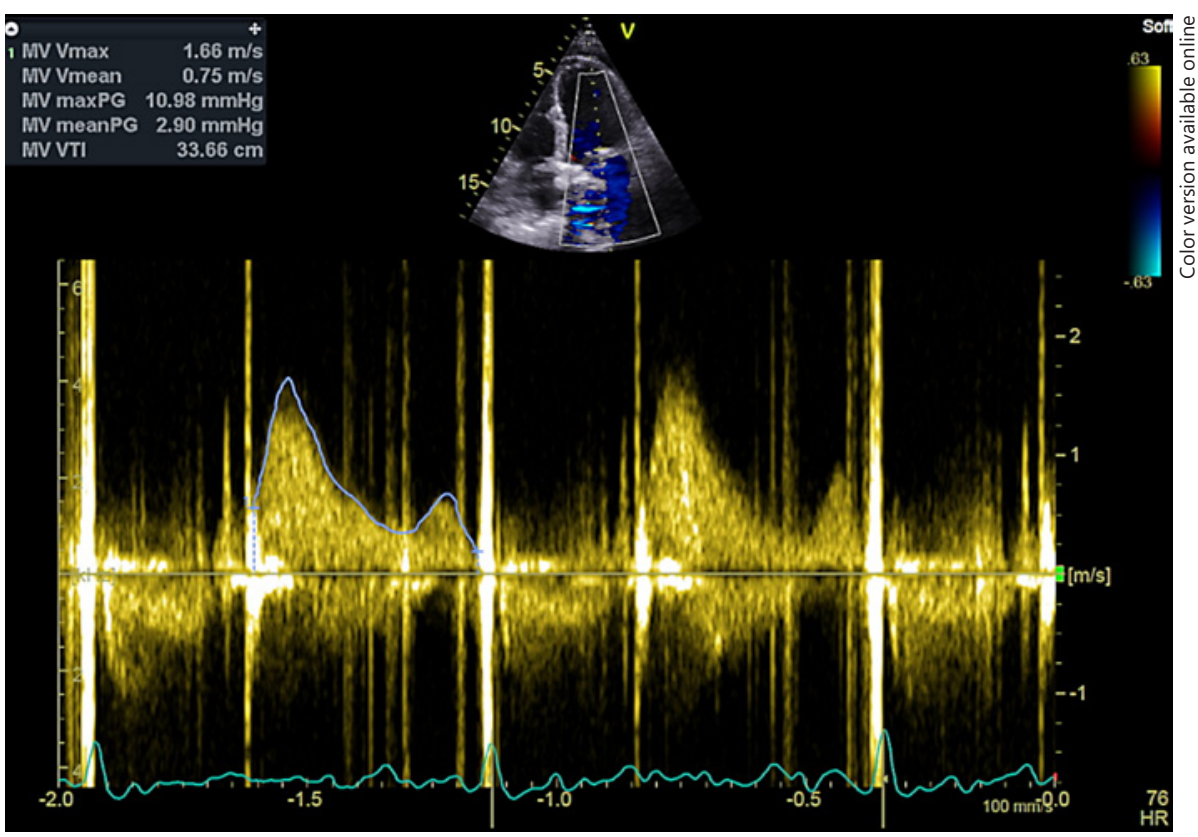

During hospitalization, he initiated therapy directed at heart failure (namely diuretics, which had to be intensified by later episodes of dyspnea, decreased peripheral oxygen saturation, and pulmonary crackles) and unfractionated heparin infusion. Four days after hospitalization, he was transferred to a center with cardiothoracic surgery to perform urgent valve replacement.

Intraoperative findings confirmed the mechanical valve thrombosis. The patient underwent a careful and delayed resection of the mitral prosthesis (Fig. 3a, b), removing all the prosthetic material, with subsequent implantation of another mechanical prosthesis, this time of the model On-x 27/29. A coronary bypass surgery with a pedicled left internal mammary artery to the anterior descending coronary artery was also performed. The procedure was uneventful.

The early postoperative period had no complications. The patient was discharged on warfarin therapy with therapeutic INR. The postoperative echocardiogram showed a normofunctioning mechanical mitral prosthesis (Fig. 4).

\section{Discussion}

In a patient with mechanical valvular prosthesis, recent onset or rapid worsening of heart failure should alert to an obstruction of the prosthesis, especially if the prosthesis is in the mitral position. Among the causes of mechanical valvular obstruction, the most frequent are prosthetic valve thrombosis (reported to be $0.3-1.3 \%$ patient-years), infective endocarditis ( $0.5-1 \%$ patients-years), and pannus $[2,3]$. Thrombosis and endocarditis may present with acute or more drawn manifestations, depending on the speed of installation. The pannus usually presents with chronic evolution, over several weeks or months [2].

The clinical presentation seems more suggestive of prosthesis thrombosis or infective endocarditis. The first hypothesis is corroborated by the previous history of warfarin suspension and its substitution by a direct anticoagulant, which is considered ineffective in this clinical scenario and therefore is contraindicated [4]. The thrombotic process may have been subacute (over the last 6 months), having become obstructive in the recent past (1 week).

The elevation of CRP should raise the suspicion of infective endocarditis. However, blood cultures were all negative. Fever has been reported in cases of prosthesis thrombosis without endocarditis [3], so one can assume that there can be an inflammatory process only because of the thrombosis per se.

The occurrence of prolonged chest pain 1 week before, together with the onset of heart failure, also raises the suspicion of an acute coronary syndrome. The documentation of obstructive atherosclerotic coronary artery disease, together with the embolic source, makes its occurrence very likely. Even though the troponin I was within the NR, there had been a long period since the beginning of chest pain. On the other hand, it seems unlikely that an 
acute coronary syndrome was the cause of heart failure, being probably a complication and not the preponderant cause of the clinical picture.

The only randomized study including patients with mechanical valvular prosthesis comparing the use of warfarin with a direct anticoagulant was RE-ALIGN, which was suspended prematurely due to the occurrence of an excess of thromboembolic and hemorrhagic phenomena in the arm treated with dabigatran. In this study, the dose of dabigatran used was significantly higher than the doses approved for use in patients with nonvalvular atrial fibrillation [5].

After research in the literature, only one mechanical thrombosis associated with the use of rivaroxaban was described, but contrary to this report, in the previously published case the mechanical prosthesis was in the aortic position, and the patient had a fatal outcome [6].

Recently, a Brazilian group published a study with a series of patients with mechanical valve prostheses implanted $>3$ months ago and with an INR described as labile, in whom the vitamin $\mathrm{K}$ antagonists were replaced by direct anticoagulants, allegedly without occurrence of thrombotic or hemorrhagic complications in any of the patients [7]. This study, however, presents obvious methodological limitations: (a) non-randomized patients were selected; (b) the study has no control group; (c) the series is very small; and (d) the follow-up is short. For this reason, these observations would require validation in largescale, randomized, controlled trials.

\section{Acknowledgement}

We would like to thank Dr. Jorge Mimoso who helped obtaining the patient's informed consent. We would like to thank the municipality of Loule for supporting the publication of this paper.

\section{Statement of Ethics}

The patient gave his written informed consent to publish his case (including publication of images).

\section{Disclosure Statement}

The authors have no conflicts of interest to declare.

\section{Funding Sources}

The authors did not receive any funding.

\section{Author Contributions}

The corresponding author, Daniela Marisa Carvalho Silva, has written the document, which was then revised by all other authors.

Coauthors Ana Braga and José Neves performed heart surgery and were responsible for gathering a big amount of clinical information, as well as some of the pictures.

Coauthor Ilídio de Jesus coordinated the patient's management and the writing process.

\section{References}

1 European Medicines Agency - European public assessment report for Xarelto [Internet]. Available from: http://www.ema.europa.eu/ docs/en_GB/document_library/EPAR_-_ Product_Information/human/000944/ WC500057108.pdf.

2 Tasca R, Tasca M, Amorim P. Evaluation of Prosthetic Valves with Echocardiography and Doppler Ultrasound. Rev Bras Ecocardiogr Imagem Cardiovasc. 2011;24(3):58-83.
3 Roudaut R, Serri K, Lafitte S. Thrombosis of prosthetic heart valves: diagnosis and therapeutic considerations. Heart. 2007 Jan;93(1): $137-42$.

4 Baumgartner H, Falk V, Bax JJ, De Bonis M, Hamm C, Holm PJ, et al.; ESC Scientific Document Group. 2017 ESC/EACTS Guidelines for the management of valvular heart disease. Eur Heart J. 2017 Sep;38(36):2739-91.

5 Eikelboom JW, Connolly SJ, Brueckmann M, Granger CB, Kappetein AP, Mack MJ, et al.; RE-ALIGN Investigators. Dabigatran versus warfarin in patients with mechanical heart valves. N Engl J Med. 2013 Sep;369(13):120614.
6 Kumar V, Kelly S, Raizada A, Yee J, Anuwatworn A, Stys A, et al. Mechanical valve thrombosis on rivaroxaban: are novel anticoagulants really an option? Methodist DeBakey Cardiovasc J. 2017 Apr-Jun;13(2):73-5.

7 Durães AR, Bitar YS, Lima ML, Santos CC Schonhofen IS, Filho JA, et al. Usefulness and safety of rivaroxaban in patients following isolated mitral valve replacement with a mechanical prosthesis. Am J Cardiol. 2018 Sep; 122(6):1047-50. 